\title{
Discurso y política indígena en el Oncenio de Leguía
}

\author{
Discourse and indigenous policy during Leguia's Oncenio
}

\author{
Discurso e política indígena no Oncenio de Légua \\ Américo Gonza Castillo ${ }^{1}$ \\ Universidad Nacional Mayor de San Marcos \\ americounmsm@gmail.com \\ Código ORCID: https://orcid.org/0000-0002-9579-1129
}

\begin{abstract}
RESUMEN
El presente artículo aborda el discurso y la política indígena dirigido a la población indígena por parte de Augusto B. Leguía y las acciones emprendidas desde el aparato estatal por dicho mandatario, en su gobierno de 1919 a 1930, denominado el Oncenio. A través de la revisión de fuentes primarías, las cuales se consultaron en el Archivo General de la Nación, y del análisis documental respecto al tema planteado, se evidencio que las acciones, emprendidas por el presidente Leguía, con respecto a la problemática que atravesaban los ciudadanos miembros de los pueblos originarios, fueron de corte populista y que en la práctica la política indígena, en el Oncenio, no tuvo un impacto significativo en la mejora de las condiciones de vida de aquella población.
\end{abstract}

\begin{abstract}
This article looks into August B. Leguia's political discourse on indigenous issues and the actions he undertook from the State apparatus during his 1919-1930 administration - the so-called Oncenio (the Elevenium). The primary sources reviewed at the Archivo General de la Nación (The Nation's Archives) and the document analysis performed evidenced that Leguia's political discourse was populistic and that the actions undertaken by his administration meant little to the well-being of indigenous populations.
\end{abstract}

\section{RESUMO}

Este artigo estuda o discurso e a política dirigida à população indígena por Augusto B. Leguía e as ações empreendidas pelo aparelho de estado do referido presidente, em seu governo de 1919 a 1930, denominado Oncenio. Através da revisão de fontes primárias, as quais foram consultadas no Arquivo Geral da Nação (Peru), e da análise bibliográfica sobre o tema levantado, constatou-se que as ações, empreendidas pelo Presidente Leguía, sobre o problema que enfrentavam os cidadãos, membros dos povos indígenas, eram de natureza populista e que na prática a política indígena, no Oncenio, não teve um impacto significativo na melhoria das condições de vida dessa população.

PALABRAS CLAVE: población indígena; Oncenio; indigenismo; política indígena.

KEYWORDS: indigenous populations; Oncenio; indigenism; indigenous politics.

PALAVRAS-CHAVE: população indígena; Oncenio; indigenismo; política indígena.

1 Historiador, abogado, egresado de la maestría en gestión de políticas pública, servidor público en el Despacho Presidencial.

Citar como:

Gonza, A. (2020). Discurso y política indígena en el Oncenio de Leguía. Espiral, revista de geografías y ciencias sociales, 2(3), 069 - 076. http://dx.doi.org/10.15381/espiral.v2i3.17731 


\section{Introducción}

El 4 de abril de 1919, Augusto Bernardino Leguía y Salcedo gano las elecciones presidenciales; sin embargo, el 4 de julio del mismo año, días antes de acercarse la fecha establecida para juramentar al cargo, encabezo un golpe de Estado y asumió de facto la Presidencia, aduciendo que sus adversarios políticos pretendían desconocer la voluntad del pueblo expresada en los resultados, del proceso electoral, que él consideraba había ganado. Después de tomar el poder, Leguía convoco a una Asamblea Constituyente que redacto una nueva constitución; así mismo dentro de esta asamblea se conformó una comisión que realizó el recuento de los votos. Aquella comisión concluyo que, en efecto, Leguía había ganado las elecciones, validando de esa manera su Presidencia.

A pocos días de haber cumplido cien años de aquellos históricos acontecimientos que marcaron el inicio del denominado "Oncenio de Leguía" se hace una remembranza de este periodo, el cual ha generado y aún sigue generando, muchas pasiones y contradicciones, en nuestro país. Del Oncenio, son muchos y amplios los elementos que se pueden estudiar; no obstante, en este artículo solo abordaremos el discurso y la política indígena, efectuada por el presidente Leguía, considerando que las demandas de los pueblos originarios son una problemática vigente hasta nuestros días. Estas demandas salen a relucir con los constantes conflictos sociales que se presentan a lo largo y ancho de nuestro territorio nacional (Ministerio de Cultura [MINCU], 2015). Los conflictos generados por la ejecución de los proyectos mineros "Las Bambas" en Apurímac, "Tía María” en Arequipa y "Quellaveco" en Moquegua que involucran a las comunidades campesinas, de aquellas regiones, con las empresas extractivas y el Estado, son algunos de los más recientes.

Debe señalarse, que luego de lograda nuestra Independencia, la población originaria de nuestra patria fue ignorada del ordenamiento jurídico administrativo y demás instrumentos normativos que se emanaron por parte de la administración pública. Así mismo, no fue reconocida la existencia, de las organizaciones indígenas, en las primeras ocho constituciones promulgadas en el país. Hubo que transcurrir un siglo para que se les reconociera su existencia y sus derechos sobre sus tierras y esto sucedió en la Constitución de 1920, promulgada por Leguía.

Puesto que, en la República continuo con la tradición colonial opresiva hacia la población indígena, muchos intelectuales reaccionaron e hicieron suyas sus demandas. En su discurso político electoral Leguía encauso la problemática indígena, las demandas de las juventudes, de los universitarios, de la clase obrera y de otros sectores sociales, que se encontraban descontentos con el sector dirigente de país, de esa manera sumo adhesiones que lo condujeron a ganar las elecciones, por segunda vez en 1919. Cabe mencionar que su primer periodo de gobierno fue entre 1908 a 1912.

La exclusión del desarrollo nacional de nuestros conciudadanos originarios, en el primer siglo de vida independiente, fue un acto deplorable llevado a cabo por los grupos de poder y el sector dirigente del país, situación que el indigenismo pretendía revertir. Acorde con estos planteamientos la política indígena, desde el aparato estatal, seguida por el presidente Leguía, en el Oncenio, pretendía reivindicar la marginación que sufría la población originaria; pero solamente fue un discurso que no se llevó a la práctica.

Para contextualizar el discurso y la política indígena asumida por el presidente Leguía, es pertinente hacer una reseña de la problemática que atravesó la población originaria, entre la Independencia y el Centenario, en los primeros cien años de vida independiente. Seguidamente trataremos el surgimiento del indigenismo; y finalizaremos abordando el discurso y la política indígena emprendida por Leguía, desde el aparato estatal, en el Oncenio. 


\section{La problemática indígena entre la Independencia y el Centenario}

La configuración inicial de la política republicana en el Perú fue un proceso complejo lleno de contradicciones producto de la dinámica interna y la influencia de factores externos, sumados a la débil institucionalidad, a los conflictos sociales y los golpes de Estado que convulsionaron la política en las primeras décadas de vida independiente. Sobre ello el modelo político de Estado-nación excluyó a la población originaria, del proyecto nacional de desarrollo, a pesar que era la población mayoritaria y ancestral del territorio declarado por la naciente República.

Los sucesivos gobiernos, que rigieron el destino del país, ignoraron e hicieron caso omiso a las demandas de la población originaria de nuestra patria. En ese sentido, Capuñay (1951) enfatizó:

Los próceres nos legaron una patria. Pero de ella debían apoderarse, quienes ni siquiera ayudaron a fundarla. Los herederos de las "encomiendas" y de los "repartimientos" han de ser los amos del país. Ellos han de llegar a constituir un poder, que no reconoce derechos populares. Ellos son los que basados en su poderío económico han de tentar el poder político y con él todas las prerrogativas y privilegios que sus padres tuvieron durante el virreinato.

De tal manera, se puede afirmar, la República por obra de los hijos de los conquistadores, de los encomenderos y de los oidores, no sería sino la prolongación de la colonia.

La misma mentalidad iguales usos y costumbres conservadores métodos. Solo que se cambian términos. Las encomiendas se llamarán haciendas. El virrey presidente. (p. 124).

Por su parte, Cotler (1978) refiere:

El proceso seguido por el Estado peruano es particular en América Latina por la fuerte presencia de "la herencia colonial". Ésta tiene dos consecuencias: primero, prefigura el carácter dependiente del Perú respecto del capitalismo hegemónico, y segundo, preserva las "relaciones coloniales" en la explotación de la mayoritaria población indígena. Ambos elementos impidieron una integración plena de la sociedad, generando en la marginación tanto social como étnica e impidiendo, además, un desarrollo autónomo del Perú frente a los poderes internacionales. ( $p$. 127).

Las ideas expuestas, por los autores antes citados, muestran que los criollos descendientes de los españoles, no renunciaron al carácter colonialista de la conquista, es más, asumieron la herencia colonial como suya. En ese sentido la fundación de nuestra República, en 1821 bajo los cimientos coloniales, reprodujo el mismo sistema de opresión y exclusión que impuso la Colonia, hacia la población originaria, manteniéndose así las mismas desigualdades, que teóricamente pretendía suprimir (Capuñay, 1951, Cotler, 178).

Una evidencia clara de la exclusión que sufrió la población indígena se corrobora revisando nuestras primeras constituciones políticas donde se aprecia que en el ordenamiento constitucional, legal y administrativo del país se ignoró a nuestros conciudadanos originarios a pesar que eran la población mayoritaria, en aquella época, y cuando se les tomó en consideración fue para despojarlos de sus tierras ancestrales y utilizarlos como mano de obra barata por parte los grupos de poder económico y político, y por el propio Estado.

Sobre la base de la situación descrita, se pude sostener que la Independencia no significó la independencia de la población indígenas, ni la recuperación de sus territorios usurpados por los conquistadores españoles. Las duras condiciones de vida a la que estuvieron sometidos, en la Colonia, no mejoro en nada con el surgimiento de la República, nuestros conciudadanos originarios fueron excluidos del proyecto nacional de desarrollo, que los criollos y la oligarquía peruana concibieron como forma de gobierno. 
Por consiguiente, lo señalado en los párrafos precedentes confirma que la población indígena se encontraba desprotegida por el ordenamiento jurídico estatal y excluido de la concepción práctica y teórica del proyecto de desarrollo nacional. De tal manera que durante el primer siglo de vida independiente las comunidades nativas continuaron una existencia silenciosa, prácticamente al margen del ordenamiento legal, hecho que aprovecharon los hacendados y gamonales para expropiarles sus tierras, con la venia del Estado, puesto que, dentro de la concepción liberal de la economía, que los criollos implantaron como forma de gobierno, las organizaciones comunales y corporativas, eran concebidas como obstáculo y traba para el desarrollo nacional.

\section{Surgimiento del indigenismo}

Como ya se ha mencionado, la independencia del Perú de la Corona Española no mejoro en nada la condición de vida de la población originaria. Las duras condiciones de vida que atravesaban nuestros conciudadanos, en el siglo XIX, se acrecentaron en las primeras décadas del siglo $\mathrm{XX}$, por el encarecimiento de las condiciones de vida que conllevaron a continuas protestas de los grupos sociales excluidos, de los derechos constitucionales, que los criollos y la elite gobernante se asignaron para sí mismos. Al respecto, Capuñay (1951) afirmó:

Durante más de cien años el Perú no ha venido siendo más que un vasto feudo. La propiedad particular de unas cuantas familias. De gente que vive en Europa derrochando la fortuna que aquí hacen con el sudor, las lágrimas, el hambre, y la sangre de todo el pueblo sometido a la más abyecta condición. (p. 125)

Lo manifestado en el párrafo anterior nos muestra las duras condiciones de vida que atravesaba la población nativa del país, en aquella época. Sin asistencia de institución pública alguna, estaban expuestas a los abusos cometidos por los que ostentaban el poder económico, por los gamonales y terratenientes, que en complicidad con las autoridades políticas, religiosas y de las fuerzas del orden publico mantuvieron marginados de los derechos republicanos, a los ciudadanos originarios de nuestra patria, desde la Independencia. Todo ello configuró el surgimiento del Indigenismo como un movimiento que trato de rescatar del orden injusto a los pueblos originarios.

Ahora bien, sobre el origen del Indigenismo no existe un consenso establecido; no obstante, podemos afirmar que el indigenismo, en tanto reflexión y movimiento intelectual, se manifestó después del fin de la Guerra con Chile, cuando se cuestiona la existencia real de la nación y se plantea el lugar que los indígenas ocupan en la sociedad peruana. Este cuestionamiento, que se le hizo, al rol del Estado y a los gobernantes, origino que diversos sectores de la sociedad peruana: intelectuales, artistas, la sociedad civil organizada y algunos sectores políticos tomaran conciencia de la problemática indígena y se pronunciaran para revertir esta situación. Así, algunos intelectuales indigenistas entre los que se encontraban Pedro Zulen, Dora Mayer, Joaquín Capelo, entre otros, crearon la Asociación Pro-Indígena, en 1909, una institución privada destinada a brindar apoyo a la población indígena en sus reclamos frente a los abusos de los hacendados, gamonales, empresas extractivas y autoridades gubernamentales.

Cabe mencionar que el indigenismo no fue un movimiento uniforme, según Burga y Flores Galindo (1991) existieron cuatro vertientes; pero en términos generales el indigenismo constituyó un avance hacia el descubrimiento y valoración de la identidad nacional de aquellos grupos sociales que nunca tuvieron acceso al ámbito de las decisiones que influyeron sobre su propio destino (Germaná, 1982). Para revertir la histórica exclusión el movimiento indígena se identificó con sus demandas sociales, culturales y de propiedad de sus tierras y pregonando los derechos que les asisten (Iziga, 1994). Para romper el orden injusto que los había excluido del proyecto 
de desarrollo nacional el movimiento indígena realizó una serie de protestas y presión política, apoyados por los intelectuales urbanos que difundían los ideales indigenistas.

Podemos indicar, a modo de ejemplo, de los abusos que enfrentaban nuestros conciudadanos originarios que el 23 de abril de 1921, Roberto Patiño presidente de la comunidad campesina Chupaca en Huancayo, solicita al ministro de Fomento se nombre una comisión para que se investigue los hechos ocurridos en su comunidad, el 17 de abril del mismo año, donde murieron siete campesinos por orden del subprefecto Romero Ramírez. Denunciando por este hecho también al terrateniente Jesús David Deregaray, a su hermano Augusto Deregaray y a su cómplice, el párroco del lugar. Los denunciados eran también autoridades, David era alcalde y Augusto su hermano ara gobernador ${ }^{2}$.

En ese orden de ideas, el surgimiento del indigenismo fue un intento de la población indígena de convertirse en protagonistas de sus reivindicaciones, se movilizaron por la recuperación y conservación de sus territorios ancestrales, por la valorización de sus lenguas, de sus costumbres y de sus propias formas cultures, apoyados por una comunidad de intelectuales que hicieron eco de las demandas de este marginado grupo social $^{3}$. A si, en las primeras décadas del siglo $X X$ gracias al apoyo del movimiento intelectual indigenista, la población originaria, estaban en condición de conferir legitimidad a la fracción hegemónica y/o persona que aspiraba o se encontraba en el poder, lo que en efecto hicieron con Leguía (Germaná, 1982).

\section{Discurso y política indígena en el Oncenio}

El presidente Leguía empezó el Oncenio denominando a su gobierno la "Patria Nueva" mostrándose como un líder carismático que entendía las necesidades de la población y alegaba que busca soluciones pragmáticas alejándose de la tradicional forma de hacer política, rasgos característicos de los políticos de aquella época, quienes se enfrentaban en discusiones y represalias contra sus opositores, antes de buscar el bienestar general de la Nación. Al respecto, Capuñay (1952) afirmó que: "Leguía propugnaba un programa de concordia y perdón, y hacer un gobierno no de partido, sino nacional, al que tendrían acceso todos los hombres capaces sin distinción de credo alguno" (p. 64).

\section{Asimismo, Capuñay acotó:}

Don Augusto llamaba a los politicos y los ciudadanos que animados de buena voluntad desearan colaborar, dentro del orden, en las tareas del poder. "No aliento odios contra nadie porque creo no tener enemigos. Desde mi iniciación en la vida pública nunca me ha indignando el extravió de los hombres. Y si alguna vez reparo en ello, como ahora, es porque me alienta la idea de que en el fondo de toda alma siempre hay un buen corazón que conquistar y un cerebro que encausar". (1952, p. 64)

Ahora bien, como ya señalamos, la opresión por la que atravesaron los pueblos indígenas durante el periodo Colonial no cambio en nada con la independencia del Perú. El olvido y falta de atención a la población nativa por parte de los gobernantes de turnos, inspiró el nacimiento del indigenismo, como un movimiento intelectual y cultural que se reflejó en el ámbito político. Así, el indigenismo, que pretendía a portar soluciones a la problemática indígena fue captado y adoptado por Augusto B. Leguía, en su programa político la "Patria Nueva" mediante un discurso de reivindicación y reconocimientos de los derechos, derechos que hasta aquella época, les habían sido negados a los pueblos originarios del país.

2 Archivo General de la Nación (en adelante A.G.N), Sección de Asuntos Indígenas, Resoluciones Supremas, Leg. 3.13.2.8, año 1921, f. 02.

3 Grupo social marginado, son aquellos que no tiene acceso al ámbito de las decisiones que influyen sobre su propio destino (Germaná, 1982). 
En tal sentido, entre las principales medidas que adopto Leguía en el marco de su política de reivindicaron indígena, fue otorgar el reconocimiento jurídico, a las comunidades nativas, en la Constitución Política de 1920. Así tenemos que en los artículos $41^{\circ}$ y $58^{\circ}$ de la referida Constitución se prescribía, lo siguiente:

Art. $41^{\circ}$.- Los bienes de propiedad del Estado, de instituciones públicas y de las comunidades de indígenas son imprescriptibles y sólo podrán transferirse mediante título público, en los casos y en la forma que establezca la ley.

Art. 58 .- El Estado protegerá a la raza indígena y dictará leyes especiales para su desarrollo y cultura en armonía con sus necesidades. La Nación reconoce la existencia legal de las comunidades de indígenas y la ley declarará los derechos que les correspondan.

Además, con la finalidad de atender y tramitar los reclamos presentados por la población indígena ante una instancia pública, mediante Resolución Suprema No $02^{4}$, emitida el 12 de septiembre de 1921, Leguía crea la Sección de Asuntos Indígenas, adscrita al Ministerio de Fomento. A esta dependencia pública a partir de 1923 se le asigno un presupuesto propio. Seguidamente mediante Resolución Suprema $\mathrm{N}^{\mathrm{o}} 06^{5}$, emitida el 16 de diciembre de 1921, se nombra como jefe de dicha Sección de Asuntos Indígenas, al señor Hildebrando Castro Pozo, un destacado intelectual, miembro del movimiento indigenista.

Más adelante, el 21 de mayo de 1922, mediante Decreto Supremo $\mathrm{N}^{0} 04^{6}$ se creó el Patronato de la Raza Indígena, en cuyo contenido podemos leer lo siguiente:

El presidente de la república considerando: Que los conflictos que se vienen suscitando entre las comunidades de la sierra y los dueños y conductores de fundos colindantes, adquieren carácter de gravedad y se convierten en lucha de razas, que es preciso evitar para el normal funcionamiento de los centros industriales de aquella región. Que con este fin se hacen necesario, crear en diferentes centros de población indígena, instituciones con carácter oficial, que en cooperación a la acción que por Decreto del 12 de septiembre de $1921^{7}$, viene realizando el gobierno a favor de esta raza. De conformidad con el art. 58 de la constitución, Decreta:

Articulo 1.- Créese el Patronato de la Raza Indígena, cuyo objeto será organizar en todos los lugares de la república, donde sea necesario la protección y la defensa de ella; así como estimular por los medios más adecuados su desenvolvimiento cultural y económico.

Sucesivamente se nombraron comisiones para investigar las revueltas campesinas y los problemas sociales, surgidos por el abuso de los terratenientes, que provocaron muchos levantamientos en el campo, tal es el caso de la Resolución Suprema No $03^{8}$, emitida el 25 de marzo de 1922, donde se comisiono el Ing. Don José Fluchert, para efectuar el deslindé de tierras entre la hacienda Parco y las comunidades campesinas de Pihuas, Retama, Pacchas y Callhuas, del distrito de Huánuco. En esa fecha, Huánuco aun no era departamento.

El discurso político sobre la problemática indígena, por parte de Leguía en el Oncenio, tuvo un matiz paternalista y populista con lo cual atrajo las simpatías de la población indígena y de los intelectuales indigenistas. En efecto, podemos mencionar Resolución Suprema $N^{o}$ 03, emitida el 16 de setiembre de 1921, mediante la cual se faculto al Ministerio de Fomento para conceder pasaje de tercera a los ciudadanos indígenas que asistieron a un Congreso que se realizó en la capital. Igualmente, mediante Resolución Suprema $\mathrm{N}^{\circ} 01^{9}$, emitida el 15 de enero 1922 se faculto al Ministerio de Fomento para

4 A.G.N., Sección de Asuntos Indígenas, Resoluciones Supremas, Leg. 3.13.2.8, año 1921, f. 06.

5 A.G.N., Sección de Asuntos Indígenas, Resoluciones Supremas, Leg. 3.13.2.8, año 1921, f. 12.

6 A.G.N., Sección de Asuntos Indígenas, Resoluciones Supremas, Leg. 3.13.2.8, año 1922, f. 26.

7 Se refiere a la creación de la Sección de Asuntos Indígenas, en el Ministerio de Fomento, una institución que debía recibir los reclamos de nuestros conciudadanos indígenas.

8 A.G.N., Sección de Asuntos Indígenas, Resoluciones Supremas, Leg. 3.13.2.8, año 1922, f. 14.

9 A.G.N., Sección de Asuntos Indígenas, Resoluciones Supremas, Leg. 3.13.2.8, año 1922, f. 09. 
conceder pasaje de regreso a los comuneros que han venido a exponer sus quejas a esta capital, debiéndose cargar estos egresos al ramo de la partida para extraordinarios del Presupuesto General de la República, señala la referida Resolución.

Al formular, desde el aparato estatal, medidas para atender la problemática indígena, Leguía obtuvo su adhesión (Cotler, 2006). La población originaria que desde siempre estuvieron olvidadas, ahora si se sintieron atendidas, se sintieron identificadas con su gobierno y lo apoyaron, al hacerlo legitimaron sus actos (Germaná, 1982). Así Leguía respondió favorablemente a las demandas de la población indígena y a los intelectuales urbanos del movimiento indigenista.

Una muestra del discurso populista empleado por Leguía es que muchas veces utilizo el título de Viracocha para sí mismo, llegando incluso a pronunciar discursos en quechua, idioma que desconocía por completo (Kapsoli y Reátegui, 1977). Sobre el particular, Ionescu y Gellner (1970) señalan que el populismo es un arma organizacional para sincronizar intereses de un grupo social patrocinada por un líder, dotado de elementos carismáticos, con un discurso político que canaliza los descontentos de ciertos grupos sociales para obtener de ellos respaldo a su gobierno a cambio de darles solución a sus necesidades y reclamos, que en muchos casos no tienen un final feliz.

En efecto, en correlación a lo señalado en el párrafo anterior, no obstante, que propugnaba la protección y reivindicación de la "raza indígena", en 1920, Leguía promulgo la Ley de Conscripción Vial, que obligaba a los campesinos (mayormente indígenas) a trabajar gratuitamente en la construcción de rutas y vías de comunicación terrestre, a nivel nacional. En cierta manera revivía la institución colonial de la Mita, trabajo gratuito obligatorio para de la población indígena, para la Corona Española (Cotler, 1978). Los hacendados y las autoridades republicanas tenían todo el poder para obligar a trabajar gratuitamente a los indígenas, en la construcción de carreteras, que favorecían sus intereses económicos.

Como ya hemos mencionado para llevar a la práctica el discurso de reivindicación indígena Leguía implemento, legisló y reglamento la vida de la población indígena en campo económico, administrativo, jurídico y social, al mismo tiempo que intento resolver, sin éxito, sus históricas demandas; puesto que, si bien es cierto el Oncenio de Leguía empezó desarrollando una era de prosperidad y de crecimiento económico, que no pudo sostener por mucho tiempo, envase a empréstitos otorgados por empresas y gobiernos extranjeros sobre todo Norte Americanos. Al respecto, Klarén afirma "las buenas intenciones del gobierno de Leguía no pudieron sostenerse en el tiempo y en 1924, poco después de reelegirse, su discurso indigenista oficial declino" (2004, p. 309).

Consecuentemente, sobre la base de las ideas expuestas, las políticas públicas destinadas a reconocer derechos y satisfacer sus demandas a la población indígena, por parte de Leguía en el Oncenio, se mantuvieron sin alcanzar su objetivo hasta su reelección en el año de 1924, luego del cual se paralizo todo intento de protección y reivindicación de la marginada masa social indígena y el estatus quo volvió a imperar en el país, reprimiéndose con crueldad cualquier intento de protesta o manifestación, de la excluida y oprimida población indígena (Klarén, 2004).

\section{Reflexiones finales}

La Independencia del Perú y la fundación de la República, para la población indígena, no significo en nada, mejoras en sus condiciones de vida, solo fue un cambio de poder político donde se pasó de los amos españoles a los gobernantes criollos, que siguieron manteniendo las mismas condiciones de opresión contra nuestros conciudadanos originarios. 
Los comunidades indígenas fueron ignoradas del proyecto de desarrollo nacional y excluidas de ordenamiento jurídico administrativo del país, su reconocimiento constitucional recién se llevó a cabo en el Oncenio de Leguía, en la Constitución de 1920 promulgada por dicho gobernante.

El indigenismo pretendía sacar del orden injusto que el Estado y los gobiernos de turno les impusieron, a nuestros conciudadanos originarios, durante los cien primeros años de vida independiente, puesto que la República se fundó sobre las mismas estructuras económicas y sociales de la Colonia.

Leguía encauso en su programa político la "Patria Nueva", el descontento de los sectores sociales del país, tales como la población indígena, la clase obrera, los jóvenes y universitarios través de un discurso populista que aprovecho para ser elegido presidente en 1919.

El discurso y política indígena emprendida por Leguía, a inicios del Oncenio, no perduro en el tiempo, toda vez que luego de ser reelegido en 1924 olvido el discurso indigenista oficial, de tal manera que las condiciones de exclusión y opresión que atravesaba la población indígena continúo sin alteraciones.

\section{Fuentes}

Archivo General de la Nación. Perú, Lima.

\section{Referencias}

Burga, M. y Flores Galindo, A. (1991). Apogeo y crisis de la República Aristocrática. Perú, Lima: Ed. Rikchay Perú.

Capuñay, M. (1952). Leguía. Perú, Lima: Compañía de Impresiones y Publicaciones.

Cotler, J. (2006). Clase, Estado y Nación en el Perú. Perú, Lima: IEP.

Germaná, C. (1982). El análisis sociológico del proceso político y del Estado en el Perú: un balance de la sociología política. I Congreso Peruano de Sociología, Perú, Huacho.

Ionescu, G. y Gellner, E. (1970). Populismo sus significados y características nacionales. Argentina, Buenos Aires: Amorrortu editores.

Iziga, R. (1994). Perú: Sociología, clases sociales y sociedad. Perú, Lima: Ed. UNMSM.

Kapsoli, W. y Reátegui, W. (1987). El Campesinado Peruano. 1919-1930. Perú, Lima: UNMSM.

Klarén, P. (2004). Nación y sociedad en la Historia del Perú. Perú, Lima: IEP.

Ministerio de Cultura (2015). Política Nacional para la Transversalización del Enfoque Intercultural. Perú, Lima: Autor. 